\title{
Comments on Unified Dark Energy and Dark Matter from a Scalar Field Different from Quintessence
}

\author{
Luis P. Chimento and Mónica Forte*
}

Departamento de Física, Facultad de Ciencias Exactas y Naturales, Universidad de Buenos Aires, Ciudad Universitaria, Pabellón I, 1428 Buenos Aires, Argentina

\begin{abstract}
In a recent paper by C. Gao, M. Kunz, A. Liddle and D. Parkinson [1] the unification of dark matter and dark energy was explored within a theory, which they say is not of the Lagrangian type, containing a scalar field. This scalar field, different from the classic quintessence, can be obtained from the scalar field representation of an interacting twofluid mixture described in the paper by L.P. Chimento and M. Forte [2]. The corresponding Lagrangian of the model is just the equation (7).
\end{abstract}

Keywords: Unified models, quintessence, interaction, dark sector.

\section{INTRODUCTION}

Due to the additivity of the stress-energy tensor it is possible to describe the quintessence field in terms of two interacting fluids, namely, stiff matter and vacuum energy. Besides, the total energy-momentum conservation is equivalent to the Klein-Gordon equation [3, 4]. This description was generalized assuming a mixture of two fluids with constant equations of state, which interact between them in a flat Friedmann-Robertson-Walker model [2]. There, a scalar field $\phi$ representation of the mixture of two interacting fluids was introduced by imposing the condition that $\dot{\phi}^{2}$ is an appropriate linear combination of the energy densities $\rho_{1}$ and $\rho_{2}$ of both fluids. Under these conditions, the resulting model was called exotic quintessence.

\section{EXOTIC QUINTESSENCE}

We present the exotic quintessence model developed in [2] including two extra matter components, with energy densities $\rho_{3}$ and $\rho_{4}$, which separately satisfy their own equations of conservation. In this case, the Einstein equations read:

$3 H^{2}=\rho_{1}+\rho_{2}+\rho_{3}+\rho_{4}$,

$\dot{\rho}_{1}+\dot{\rho}_{2}+3 H\left[\left(1+w_{1}\right) \rho_{1}+\left(1+w_{2}\right) \rho_{2}\right]=0$,

$\dot{\rho}_{3}+3 H\left(1+w_{3}\right) \rho_{3}=0$,

$\dot{\rho}_{4}+3 H\left(1+w_{4}\right) \rho_{4}=0$,

where we have adopted constant equations of state $w_{n}=p_{n} / \rho_{n}$ for each fluid with $n=1,2,3,4$. Units are

*Address correspondence to this author at the Departamento de Fsica, Facultad de Ciencias Exactas y Naturales, Universidad de Buenos Aires, Ciudad Universitaria, Pabellón I, 1428 Buenos Aires, Argentina;

Tel: 54-11-4576-3390, Ext. 830; Fax: 54-11-4576-3357;

E-mail: forte.monica@gmail.com chosen so that the gravitational constant is set to $8 \pi G=1$ and $c=1$. We introduce a scalar field $\phi$ representation of the interacting two first fluids associating $\dot{\phi}^{2}$ with the following linear combination of $\rho_{1}$ and $\rho_{2}$

$\dot{\phi}^{2}=\left(1+w_{1}\right) \rho_{1}+\left(1+w_{2}\right) \rho_{2}$.

From Eqs. (2) and (5) we obtain the total energy density and pressure of the four-fluid mixture, and the dynamical equation for the scalar field

$$
\begin{aligned}
& \rho=\frac{\dot{\phi}^{2}}{1+w_{1}}+\frac{w_{1}-w_{2}}{1+w_{1}} \rho_{2}+\rho_{3}+\rho_{4}, \\
& p=\frac{w_{1} \dot{\phi}^{2}}{1+w_{1}}-\frac{w_{1}-w_{2}}{1+w_{1}} \rho_{2}+w_{3} \rho_{3}+w_{4} \rho_{4}, \\
& \ddot{\phi}+\frac{3}{2}\left(1+w_{1}\right) H \dot{\phi}+\frac{w_{1}-w_{2}}{2} \frac{\dot{\rho}_{2}}{\dot{\phi}}=0 .
\end{aligned}
$$

These equations define the exotic quintessence model.

\section{CONCLUSIONS}

Choosing $w_{1}=0, w_{3}=0, w_{4}=1 / 3$, and making the substitutions $\phi \rightarrow \phi / \sqrt{2}$ and $-w_{2} \rho_{2} \rightarrow \Lambda(\phi)$, the Einstein and the exotic quintessence equations are reduced to the expressions (21)-(23) of the paper [1]. On the other hand, if we impose the integrability condition mentioned in [2]

$\dot{\rho}_{2}+A \dot{\phi} \rho_{2}=0$,

on the field equation (8) with a constant $A$, we get $\rho_{2} \propto \Lambda(\phi) \propto \exp (-A \phi)$ after integrating the Eq. (9). Then, the exotic quintessence model with the integrability condition (9) leads to an exotic scalar field driven by an exponential potential and thus to obtain the model investigated in ref. [1]. As was already pointed in ref. [2], this model is integrable and can be solved exactly. 


\section{REFERENCES}

[1] Gao C, Kunz MAR. Liddle, D Parkinson. Unified dark energy and dark matter from a scalar field different from quintessence. Phys Rev D 2010; 81: 043520 .
[2] Chimento LP, Forte M. Unified model of baryonic matter and dark components. Phys Lett B 2008; 666: pp. 205.

[3] LP Chimento. Symmetry and inflation. Phys Rev D 2002; 65: 063517.

[4] LP Chimento. Interacting fluids generating identical, dual and phantom cosmologies. Phys Lett B 2006; 633: 9.

Received: July 26, 2011

Revised: September 12, 2011

Accepted: September 15, 2011

(C) Chimento and Forte; Licensee Bentham Open

This is an open access article licensed under the terms of the Creative Commons Attribution Non-Commercial License (http://creativecommons.org/licenses/by$\mathrm{nc} / 3.0 /$ ) which permits unrestricted, non-commercial use, distribution and reproduction in any medium, provided the work is properly cited. 